\title{
Parametric Study of Air Re-entrainment within Air-Cooled Chiller Compounds
}

\author{
Bryony Watson ${ }^{1,2}$, Julien de Charentenay ${ }^{1}$ \\ ${ }^{1}$ RED Engineering Design, London, UK \\ ${ }^{2}$ Heriot-Watt University, Edinburgh, UK
}

\begin{abstract}
Air cooled chillers serving high building cooling loads such as data centre are prone to air re-entrainment. As air re-entrainment is deemed to be specific to each project, it is customary to either ignore it or allow a nominal amount based on past experience at concept stage.

This parametric study uses Computational Fluid Dynamics (CFD) to evaluate principal components affecting air re-entrainment within air-cooled chiller compounds. Results shows that air re-entrainment increases with wind speed and that at highest wind speed air re-entrainment is linearly dependent on plant compound length. These linear relationships, which are deemed to be adequate for plant compound with heat rejection density between 15 and $25 \mathrm{~kW} / \mathrm{m}^{2}$, can be applied at concept design to provide an early estimate of air re-entrainment.
\end{abstract}

\section{Introduction}

Air cooled chillers are heat rejection equipment used to meet building cooling demand. Such equipment rejects heat to the outside in order to provide cooling for the building that is used for occupant comfort cooling in occupied buildings, or equipment cooling in critical applications such as data centres. In critical applications, the heat rejection equipment is to remain operational 24/7 even under extreme ambient conditions. To meet this requirement, data centre heat rejection equipment is selected based on ASHRAE extreme wet-bulb and $\mathrm{N}=20$ years dry-bulb temperature to meet Uptime Institute requirements (Uptime Institute Professional Services, LLC, 2012). These selection criteria are more stringent than for typical occupied building due to the nature of such facilities.

Data centres have another characteristic that set them apart from traditional occupied buildings, such as office or residential buildings. These buildings are dominated by the cooling demand of the IT equipment rather than fabric load and have year-long cooling requirement. In addition, IT equipment power density, the amount of IT electrical power demand per square meter, is increasing (Brill, 2006) and data centre are becoming larger and consume significant amount of energy with data centre power demand of up to 150MW (World's Top Data Centers, 2018). The power density and power demand increases place a heavy burden on heat rejection system design that needs to cope not only with an increase in the amount of heat rejected but also with having to reject this heat within a smaller building footprint area.

Placement, wind speed and wind direction are identified as having significant effect on hot air recirculation (Khankari, 2018). Whilst such recirculation depends on the amount of heat rejected and is more likely to be observed for large installations, they also affect smaller units such as split systems when the local airflow pattern results in a local microclimate (Bruelisauer, Meggers, Saber, Li and Leibundgut, 2014).

Air-cooled chillers are particularly vulnerable to hot air recirculation and local microclimate. These systems typically tolerate short-term exposure to temperature in excess of their selection criteria. However, as these systems are based on refrigerant, they operate up to a maximum operating temperature above which they fail due to high-pressure lockout. This mode of failure is challenging as the chiller abruptly stops providing cooling to the facility, which could result in overheating and possible IT failure if the cooling capacity shortfall associated with the chiller failure is not compensated for or if several chillers are affected.

Similar hot air recirculation problems are observed in large air-cooled condensers plant serving power plants. In this application, hot air recirculation results in degradation of performance of the air-cooled condensers rather than failure. Such applications exhibit similar characteristics to critical facilities heat rejection systems such as significant heat load dissipated and space constraint and have been studied experimentally and using Computational Fluid Dynamics (CFD) with good correlation (Maulbetsch, DiFilippo and O'Hagan, 2011). These studies clearly highlight that the effect of the wind on system performance are not only associated with wind speed, but also with wind orientation particularly when the wind interacts with adjacent buildings and structures (Maulbetsch, DiFilippo and O'Hagan, 2011; Borghei and Khoshkhoo, 2010).

This paper presents a parametric study of air reentrainment on air cooled chiller plant compounds. The study uses CFD to predict the airflow and air reentrainment within the plant compound. The analysis results are extracted to investigate the possibility of and derive, rules of thumb to assess heat re-entrainment at concept design. These rules of thumb could be applied at concept design, particularly in the design of data centre heat rejection systems, to inform initial equipment 
selection with the opportunity of further refinement once the design is documented to a suitable level.

\section{Methods}

\section{CFD Analysis}

CFD modelling is recognised and applied as a tool to investigate semi-external and external spaces (CIBSE, 2015), including urban wind environment, such as described in Ercoftac (2018). The CFD simulations presented in this study are conducted using the commercial CFD software 6SigmaDCX version 11 . The commercial CFD software 6SigmaDCX is validated against a range of cases that includes natural convection, forced convection and low-rise building (Future Facilities, 2018) and has been validated against experimental measurements in complex data centre applications (Ruiz, 2015; Alissa, et al., 2015) . The CFD software solves the Navier-Stokes equations for mass, momentum and energy using a SIMPLE algorithm to steady-state with a two-equation turbulence model. A first-order upwind discretization scheme is employed to ensure stability. Air is modelled using a $1.2 \mathrm{~kg} / \mathrm{m}^{3}$ constant density fluid with a viscosity of $0.0000184 \mathrm{~kg} / \mathrm{m}$.s. Buoyancy forces are taken into using a Bousinesq approximation based on an expansivity of $0.0033 /{ }^{\circ} \mathrm{C}$.

The CFD computational domain is centred around the chillers plant compound and is extended to allow for the establishment of the ambient wind conditions within the domain prior to entering the plant compound and downwind of the plant compound.

The CFD computational domain is discretised using unstructured meshes consisting of predominantly hexahedron cells with hanging nodes ranging between 2.3 million cells for the smallest computational domain, i.e. 1x 1 chiller plant compound, and 25 million cells for the largest computational domain. The mesh generation uses the CFD software automated gridding process that relies on local grid rules attached to individual objects such as chillers, intake and discharge vents, These grid rules have been designed to capture the key phenomena associated with the object, for example grid extrusion in the direction of the flow of discharge vents. The automated gridding allows for mesh to be allocated where needed to capture flow effects. In addition, mesh refinement zones are applied around the plant compound to limit the cell size to a maximum of $100 \mathrm{~mm}$. Cell sizes are controlled to maintain a maximum aspect ratio of no more than 10 .

Meshing rules are similar for all scenario modelled. No mesh sensitivity analysis has been undertaken for this study.

Figure 1 shows the chiller plant compound arrangement, which consists of the NxN chillers laid out in a regular pattern. The chiller plant compound is parametrised using the number of chillers in one direction $\mathrm{N}$ with $\mathrm{N}$ varying between 1 and 6 . The size of the plant compound is dictated by the number of chillers, $\mathrm{N}$; the length of the chiller, L; the width of the chiller, $\mathrm{W}$; and the chiller spacing requirements for maintenance and airflow, 1 and $\mathrm{w}$, that are nominated in the chiller manufacturer documentation.

The wind scenarios considered are based on three wind speeds: calm wind scenario, $5 \mathrm{~m} / \mathrm{s}$ and $10 \mathrm{~m} / \mathrm{s}$ wind speed. The $5 \mathrm{~m} / \mathrm{s}$ and $10 \mathrm{~m} / \mathrm{s}$ wind are assessed from 3 directions: (1) along the length of the chiller compound, denoted direction $1,(2)$ at a $45^{\circ}$ angle from the length of the chiller compound, denoted direction 2 , and (3) direction along the width of the chiller compound, denoted direction 3 . These scenarios allow to assess the impact of both wind speed and wind direction that are expected to impact air re-entrainment (Ge, Xiao, Wang and $\mathrm{Pu}, 2012$, Borghei and Khoshkhoo, 2010; Khankari, 2018) albeit in a building0-free environment.

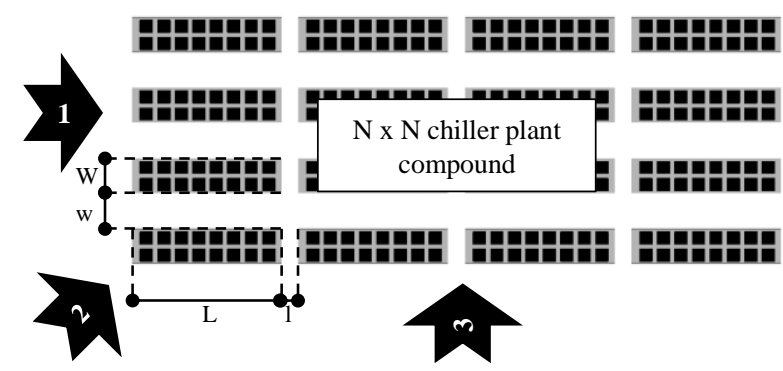

Figure 1: Plan view of CFD computational domain anotating characteristic distances and analysed wind directions.

Under calm wind scenario, the CFD model boundary conditions are based on zero pressure boundary conditions at the external and sky boundary conditions, with specified temperature where air enters the computational domain and zero temperature gradient where air exits the domain.

A $\log$ law profile based on the nominated wind speed achieved at a $10 \mathrm{~m}$ reference height is applied at the inlet boundary condition combined with a zero-gradient pressure and fixed temperature boundary conditions for the $5 \mathrm{~m} / \mathrm{s}$ and $10 \mathrm{~m} / \mathrm{s}$ wind speed scenarios. Zero-gradient boundary conditions are applied for velocity and temperature at the outlet boundary conditions together with a fixed pressure boundary condition. The sky boundary condition is set to a slip wall boundary condition to allow for the wind to remain established. The sky domain boundary height is set to allow for a ratio of chiller plant compound height over domain height of circa $5 \%$ and an overall blockage ratio of less than $1 \%$ to ensure no unrealistic acceleration of the wind environment due to constriction.

In all model, the ambient temperature is modelled as $40^{\circ} \mathrm{C}$.

\section{Air-Cooled Chiller Parameters}

The air-cooled chillers are modelled based on chiller selection sourced from two different manufacturers based on the following selection criterion:

- Ambient temperature: $40^{\circ} \mathrm{C}$;

- Chiller water fluid type: $100 \%$ water; 
- Chilled water flow/return temperature:

$23^{\circ} \mathrm{C} / 17^{\circ} \mathrm{C}$

- Chiller cooling capacity:

$$
\begin{array}{ll}
\circ & 250 \mathrm{~kW} ; \\
\circ & 750 \mathrm{~kW} ; \\
\circ & 1500 \mathrm{~kW} .
\end{array}
$$

This study uses two manufacturers to ascertain whether the choice of manufacturer would result in different amount of heat re-entrainment rather than comparison of performance, hence chiller manufacturers are anonymised. The chillers' selection from both manufacturers use different compressor and free cooling technology. The chiller selections' key parameters relevant to the study are listed in Table 3 . All chillers are $2,200 \mathrm{~mm}$ wide, but have different length, height, maximum cooling capacity, input power, number of fans and airflow. Chiller heat rejection is defined as the sum of the cooling demand, i.e. the amount of cooling to be provided by the chiller to the building, and the input power, i.e. the power used by the chiller itself to meet the cooling.

The plant compound chiller arrangement is based on the manufacturers' minimum clearance recommendations, defined as dimensions $l$ and $w$ in Figure 1 and summarised in Table 1. These recommendations are different for both manufacturers and are understood to cater for minimum space requirements for both airflow and maintenance. This study does not investigate increasing clearance space above the minimum requirements as most projects are likely to be designed to the minimum requirements. Increasing clearance space is expected to reduce air reentrainment (Khankari, 2018) and is considered an airflow management measure.

\section{Table 1: Minimum clearance requirements}

\begin{tabular}{|c|c|c|c|}
\hline Chiller & Manufacturer & $\begin{array}{c}\text { Clearance, } \\
\boldsymbol{l}\end{array}$ & $\begin{array}{c}\text { Clearance, } \\
\boldsymbol{w}\end{array}$ \\
\hline A/250kW & A & $2600 \mathrm{~mm}$ & $2600 \mathrm{~mm}$ \\
\hline A/750kW & A & $2600 \mathrm{~mm}$ & $2600 \mathrm{~mm}$ \\
\hline A/1500kW & A & $2600 \mathrm{~mm}$ & $2600 \mathrm{~mm}$ \\
\hline B/250kW & B & $1100 \mathrm{~mm}$ & $2300 \mathrm{~mm}$ \\
\hline B/750kW & B & $1100 \mathrm{~mm}$ & $2300 \mathrm{~mm}$ \\
\hline B/1500kW & B & $1100 \mathrm{~mm}$ & $2300 \mathrm{~mm}$ \\
\hline
\end{tabular}

Each chiller is represented using a black-box model as shown in Figure 2 that is based on the physical external dimensions of the chiller. The black-box model represents the chiller as a block with 2 intakes on each elongated side and discharge vents. Each discharge vent is modelled using a swirling fan model accounting for the fan diameter and fan hub diameter with a 0.3 swirl factor. The chiller airflow is equally split between the intake vents and the discharge vents. The discharge vent air temperature is based on the averaged intake air temperature raised by the chiller total heat rejection, defined as the sum of cooling capacity and input power, thereby allowing coupling of the discharge conditions to the intake conditions. This approach is similar to the approach taken in Khankari (2018) and allows for a representation of the chiller behaviour without the complexity of modelling chiller individual components, whose performance and characteristics are unknown.

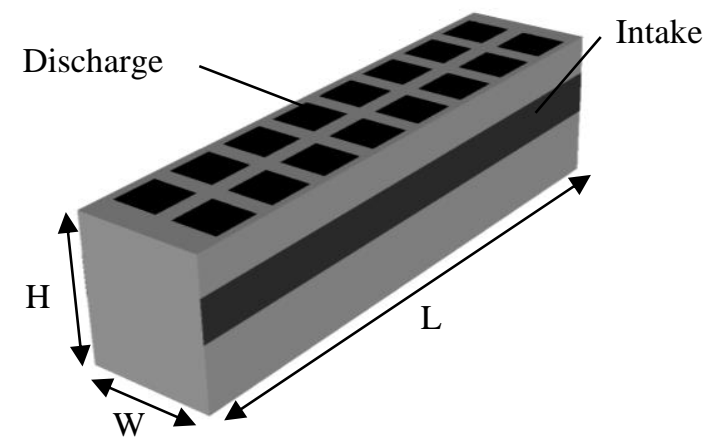

Figure 2: Chiller representation

\section{Dataset}

The CFD analysis undertaken for this study is used to define a parametric dataset. The dataset parameters are summarised in Table 2 and the dataset consists of 252 simulation results data-points.

Table 2: Dataset parameters

\begin{tabular}{|c|c|}
\hline Parameter & Values \\
\hline Manufacturer & $\mathrm{A} / \mathrm{B}$ \\
\hline Chiller cooling capacity & $250 \mathrm{~kW} / 750 \mathrm{~kW} / 1500 \mathrm{~kW}$ \\
\hline Plant compound size & $1 \times 1 / 2 \times 2 / 3 \times 3 / 4 \times 4 / 5 \times 5 /$ \\
& $6 \times 6$ \\
\hline Wind speed & Calm / 5m/s / 10m/s \\
\hline Wind direction & (1) Length-wise / (2) Diagonal \\
& $/$ (3) Width-wise \\
\hline
\end{tabular}

The dataset is employed to extract correlation between chiller plant compound parameters, aka input data vectors, and air re-circulation outcomes, aka output data vectors, predicted by the CFD simulation using statistical analysis. To allow the statistical analysis to be undertaken, the dataset is articulated around the input parameters characterising:

- Chillers: chiller length $L$, chiller width $W$, chiller heat rejection $Q_{H}$, chiller airflow $Q_{V}$, chiller temperature rise $\Delta T_{C H}$;

- Plant compound: number of chiller $N$, plant compound length $L_{P C}$, plant compound width $W_{P C}$, plant compound area $A_{P C}$, total heat rejected $Q_{H, P C}$, total airflow $Q_{V, P C}$; and

- Wind: wind speed, wind direction.

The following two output parameters characterising the hot air re-entrainment are extracted from the CFD simulation: (a) the Most Affected Chiller Intake Temperature Rise is the intake temperature rise reported at the chiller with the highest intake temperature rise and (b) the Average Chiller Intake Temperature Rise is the intake temperature rise averaged over all chillers within the plant compound.

Whilst the Most Affected Chiller Intake Temperature Rise quantifies the impact of the air re-entrainment on the most affected equipment, i.e. equipment most at risk of failure, the Average Chiller Intake Temperature Rise quantifies how air re-entrainment affects the plant compound as a 
whole and quantifies the impact of air re-entrainment on plant efficiency.

The statistical analysis of the dataset results uses principal component analysis as described in Buskirk (2018). Input and output data vectors are presented as normalised input and output data vectors. The normalisation process consists of shifting the data vector to ensure a zero mean and scaling it by the standard deviation to limit its spread as indicated in equation (1). Scaled data vectors are identified as normalised data vectors with their physical counterpart referred to without the prefix normalised.

$$
d_{i}^{*}=\frac{d_{i}-\langle d\rangle}{\sqrt{\sum_{j=1}^{N}\left(d_{j}-\langle d\rangle\right)^{2}}}
$$

Where $d_{i}^{*}$ denotes the normalised output parameter value for scenario $i, d_{i}$ denotes the unnormalised output parameter value for scenario $i,\langle d\rangle$ the average of the output parameter values across all scenarios, and $N$ the number of scenarios.

\section{Results}

The results are presented in two stages. In the first stage, the results are described to identify the key phenomena driving air re-entrainment, hereby providing insight in the interaction between the wind environment and chiller plant compound. In the second stage, the dataset is analysed using statistical methods to identify correlations.

\section{Qualitative Analysis}

Simulations are conducted for plant compound ranging from $1 \times 1$ to $6 \times 6$ chillers. Figure 3 presents the Most Affected Chiller and Chiller Average Intake Temperature Rise extracted from the analysis results for the chiller A/750kW under calm ambient wind condition. The Most Affected Chiller, located in the centre of the plant compound as shown in Figure 4, is pulling air throughout the plant compound and hence is more likely to re-entrain air that has been discharged by adjacent chillers. This effect becomes more pronounced as the number of chillers increases. The Chiller Average Intake Temperature Rise also increases with the number of chillers, albeit by a lower amount as the intake temperature increase observed at the chillers located in the centre of plant compound is compensated by the chillers located at the perimeter of the plant compound that have very low amount of air re-circulation. Similar behaviour is observed for all chillers regardless of manufacturer and size.

The local wind environment, defined by wind speed and wind direction in relation to the plant compound, has an impact on the air re-entrainment as expected and described in Khankari (2018) although with some difference due to the inclusion of a side building investigated in Khankari (2018) and the larger number of variation in chiller size and plant compound size investigated in the present study.

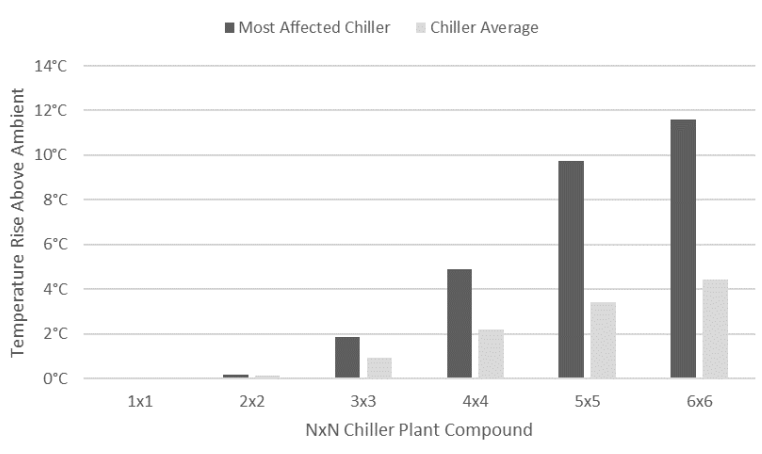

Figure 3: Air intake temperature rise above ambient for chiller $A / 750 \mathrm{~kW}$ under calm scenario.
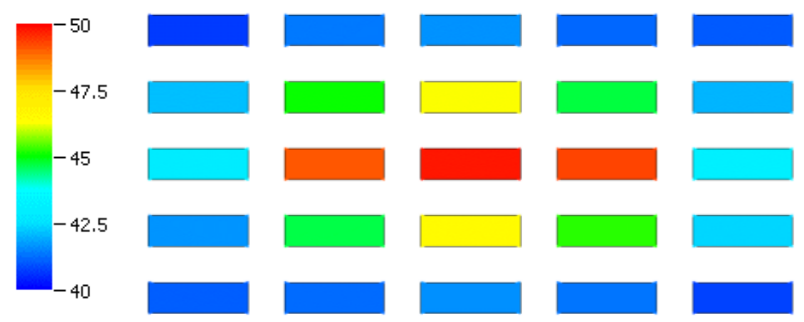

Figure 4: Chiller coloured by intake temperature for chiller A/750kW under calm scenario and $5 \times 5$ plant compound.

The wind speed generally increases the chiller air recirculation compared to calm wind environment due to the wind entraining the chiller discharged air. Whilst the Chiller Average and Most Affected Chiller Intake Temperature Rise increase with wind speed at $5 \mathrm{~m} / \mathrm{s}$ and $10 \mathrm{~m} / \mathrm{s}$ wind for plant compound of $3 \times 3$ chillers or less, the opposite generally occurs when the plant compound increases to $4 \times 4$ chillers or above.

The impact of the wind speed also varies with wind direction. Wind direction 3, where the wind is oriented perpendicular to the chillers, is generally found to result in lower overall intake air temperature than wind direction 1 and 2. Furthermore, the air intake temperature reported under this wind direction is also shown to be sensitive to the wind speed with higher wind speed resulting in lower air intake temperature when the plant compound exceeds $4 \times 4$. This observation is different from the observations made in Khankari (2018) and is attributed to the use of plant compound in $\mathrm{NxN}$ configuration rather than the $4 \mathrm{x} 1$ configuration employed in Khankari (2018). In this study, the $\mathrm{NxN}$ plant configuration limits the amount of unaffected ambient air being drawn to only the chillers located at the perimeter of the plant compound and the increased wind speed result in higher dispersion of the chiller discharge air and a reduction in intake temperature rise.

The effect of the plant compound size on chiller discharge plume is shown in Figure 9 for chiller A/1500kW under $5 \mathrm{~m} / \mathrm{s}$ wind in direction 1 . This setup is considered as it is results in high level of air re-entrainment. The figure shows the discharge of one chiller, marked using streamlines from each of the 22 fans, located at the most 
upwind and at the end of the plant compound for plant compound size ranging from $1 \times 1$ to $6 \times 6$.

The chiller discharge in the single chiller, i.e. $1 \mathrm{x} 1$ plant configuration, shows symmetric air discharge patterns with no recirculation occurring. When the plant compound size is increased, the discharge patterns are no longer symmetric, but show preferentially entrainment towards the outside of the plant compound, where the discharge shows a vortex airflow pattern. This preferential entrainment is attributed to the interaction with the adjacent chillers' discharge that displace the discharged air for the considered chiller to the outside of the plant compound. This effect becomes more pronounced when the plant compound size increases.

The streamlines indicate that the chiller discharge enters a vortex pattern that appears centred over the edge of the plant compound. It is anticipated that this vortex is formed as a combination of the following factors: interaction of the wind with the chiller's obstruction, displacement of the wind by the chiller discharge and air demand from the chiller at low level. This vortex effect starts to appear from plant compound size of $2 \times 2$ onwards. Although at this plant compound size, the downwind chillers do not appear affected by air recirculation. When the plant compound size increases to $3 \times 3$ and above, the air discharge plume vortex increases, and the discharge plume starts to affect downwind chillers with streamlines indicating that some discharge air is being re-entrained at downwind chillers. This effect is anticipated to increase by compound effect when intake air temperature increase result in discharge air temperature increase that further affect downwind chillers.

\section{Statistical Analysis}

The interaction between chillers and wind environment is complex and difficult to represent using a simplified bottom-up, physics-based model. Instead, a statistical approach is employed to represent this interaction using representative inputs characterising chiller, chiller plant compound, and wind environment and representative outputs quantifying air re-entrainment.

The analysis of the dataset's normalised input vectors shows correlation between total heat rejection over the plant compound and total airflow and plant compound length and areas. The correlations between total heat rejection $Q_{H, P C}$ and total airflow $Q_{V, P C}$ and plant compound length $L_{P C}$ are shown in Figure 5.

The correlation between plant compound total heat rejection and airflow indicates a linear relationship with little difference between manufacturers. Indeed, chiller heat rejection and airflow are related by the temperature rise across the chiller, which varies between $8.1^{\circ} \mathrm{C}$ and $12.3^{\circ} \mathrm{C}$ and is constant for each chiller model.

Plant compound total heat rejection and plant compound length are strongly coupled albeit by a non-linear relationship. The relationship between these two parameters can be explained through reasoning. The total heat rejection depends on number of chillers, $\mathrm{NxN}$, and chiller heat rejection. The chiller heat rejection is related to the length of the chiller, with heat rejection per linear meter length of chiller varying between $67 \mathrm{~kW}$ and $151 \mathrm{~kW}$ per meter length. The plant compound length depends on the number of chillers in the length direction, $\mathrm{N}$, the length of the chiller and the clearance between chiller in the lengthwise direction. Combining these two expressions make it clear that whilst the plant compound length varies linearly with the number of chiller $\mathrm{N}$, the total heat rejection varies with the square of the number of chiller $\mathrm{N}$, thereby resulting in the relationship observed.

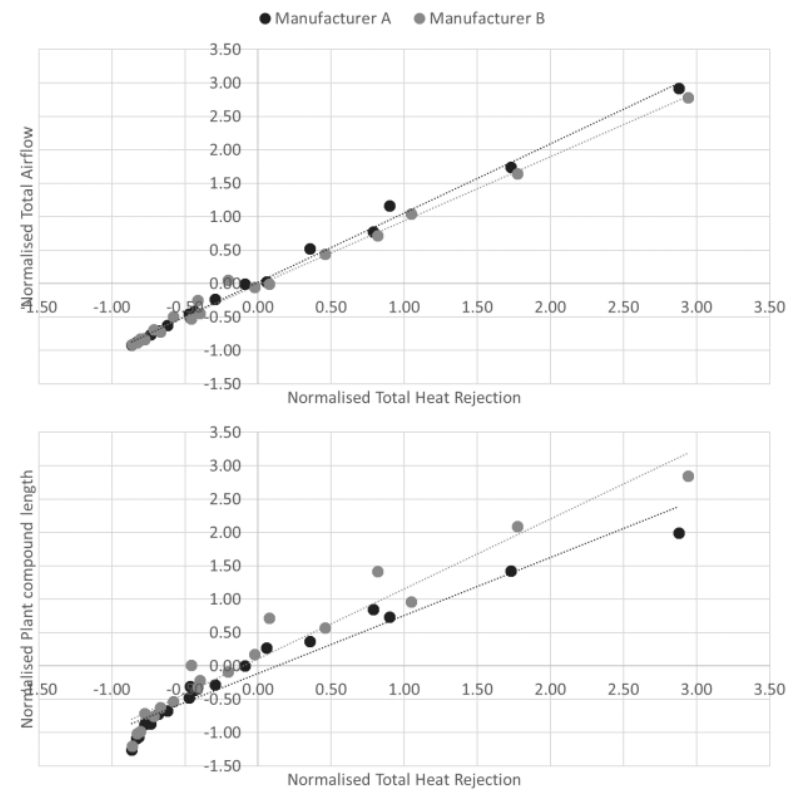

Figure 5: Correlation of total heat rejection with total airflow and plant compound length

The statistical analysis is undertaken into two steps. In the first step, a principal component analysis is undertaken to evaluate the dominant input vector parameters for each output vector parameter. In the second step, linear correlations are evaluated based on their fit, measured using R-squared or coefficient of determination.

The principal component analysis is undertaken for the whole dataset and is repeated for the Chiller Average and Most affected Chiller Intake Temperature Rise. In both cases, the principal component identifies the plant compound length $L_{P C}$ as the most dominant component, with a correlation coefficient of 0.92 and 0.89 against the Chiller Average and Most Affected Chiller Intake Temperature Rise when considering all data points. However, a plot of the dataset shows a high spread in results indicating that whilst area length is the dominant parameter it is not the sole parameter. If the area length was the sole dominant parameter, it would infer that air re-entrainment only depends on the size of the plant compound. In such case, the same level of air reentrainment would be anticipated for calm wind and $10 \mathrm{~m} / \mathrm{s}$ wind scenarios, which is not consistent with the airflow pattern observed and discussed in the previous section.

To refine the analysis, the dataset is divided to consider each wind scenario individually. This process results in 7 smaller datasets that include results from both 
manufacturers. The principal component analysis is repeated for each of the 7 datasets separately. The analysis shows that the plant compound length $L_{P C}$ remains the dominant component in all wind condition analysed, with correlation coefficients ranging between 0.91 and 0.99 . Higher correlation coefficients are reported for higher wind speed, which is taken as an indicator that as wind speed increases the impact of the difference between chillers decreases.

Figure 6 and Figure 7 plots the normalised Chiller Average and Worst Affected Chiller Intake Temperature Rise against the normalised plant compound length for the calm wind dataset and the wind direction $1,10 \mathrm{~m} / \mathrm{s}$ wind speed dataset. The latter dataset is used as this wind condition results in higher air re-entrainment.

The distribution of the data points indicates that a linear approximation of the chiller intake temperatures can be used when manufacturers are considered individually. The linear approximation of chiller air intake temperature with plant compound length provides a better fit at high wind speed, R-squared of 0.95 to 0.98 , than in the calm wind scenario, R-squared of 0.82 to 0.95 . This is attributed to differences in air re-entrainment process between calm and high-wind scenarios.

In calm wind scenarios, the air re-entrainment is driven by the air aspiration from the chillers that drives ambient air within the chiller compound and is affected by clearance spaces, chiller height for example. In high wind scenarios, the air re-entrainment is driven by the entrainment of chiller discharge by the ambient wind that can be intuited to linearly depend on the travelling air distance, namely the plant compound length for wind direction 1.

The comparison of the analysis results for both manufacturers highlights a difference in the amount of air re-entrainment in the calm wind scenario with the air reentrainment predicted for chillers of manufacturer B being lower than for manufacturer A regardless of the chiller size. This difference is attributed to chiller characteristics such as chiller height and clearance spaces that are different between the manufacturers and affects air re-entrainment in low wind only.

As the wind speed increase, the difference between the manufacturer narrows down with the $10 \mathrm{~m} / \mathrm{s}$ wind speed results showing little difference.

Using the dataset limited to the wind direction $1,10 \mathrm{~m} / \mathrm{s}$ wind speed condition, a linear interpolation is extracted for the normalised Chiller Average and Most Affected Chiller Intake Temperature Rise that is based on the data from both manufacturers combined. The dataset and linear interpolation are shown in Figure 8, which indicates nearly identical linear interpolation coefficients for the Chiller Average and Most affected Chiller Intake Temperature Rise. Thus, indicating that both output vectors are affected similarly, albeit with different magnitude.

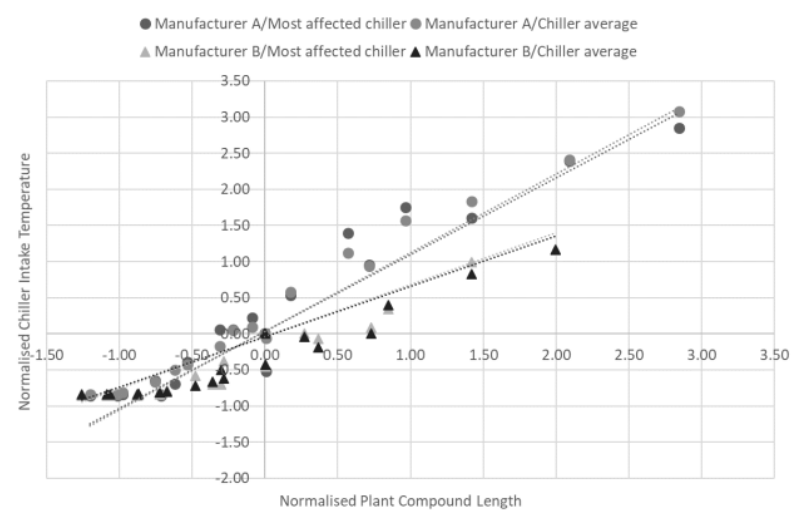

Figure 6: Correlation between normalised chiller intake temperature and normalised plant compound length under calm wind conditions

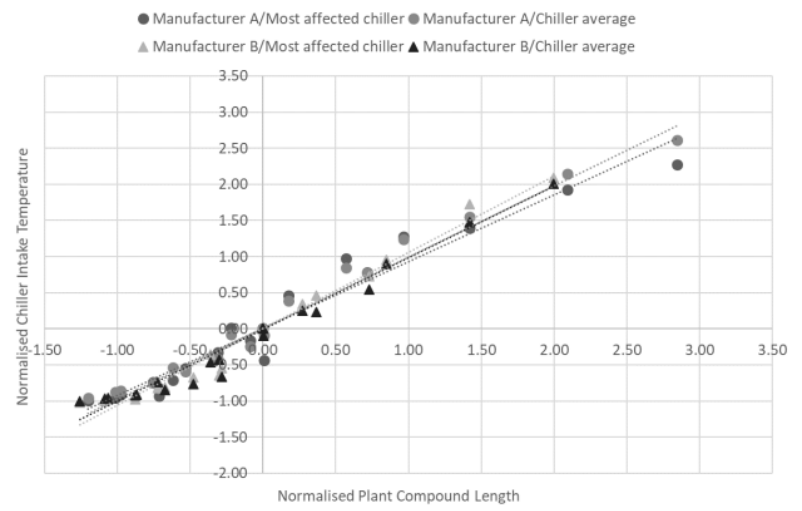

Figure 7: Correlation between normalised chiller intake temperature and normalised plant compound length under wind direction 1 and wind speed of $10 \mathrm{~m} / \mathrm{s}$

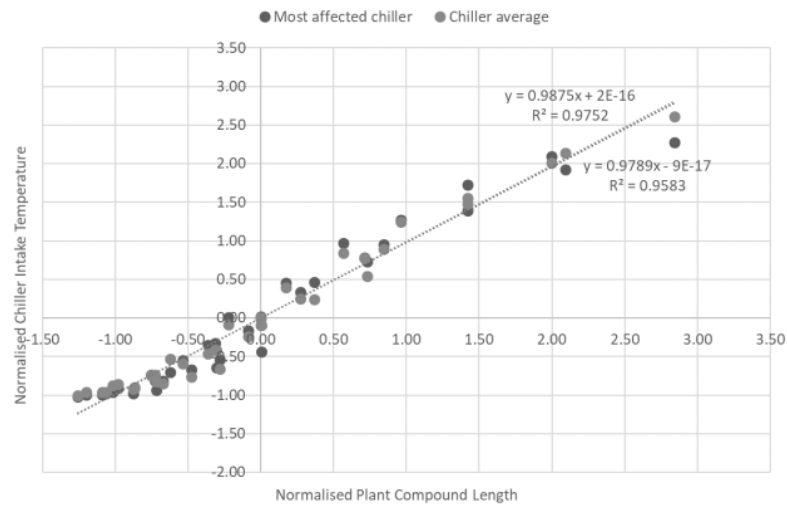

Figure 8: Correlation between normalised chiller intake temperature and normalised plant compound length under wind direction 1 and wind speed of $10 \mathrm{~m} / \mathrm{s}$ considering a dataset composed of both manufacturer results.

The linear interpolation can be rewritten as the following estimates:

- Chiller Average Temperature Rise estimate:

$$
\Delta T=8.7 \max \left(\frac{L_{P C}-38.9}{26.8},-1\right)+9.0
$$

- Most Affected Chiller Temperature Rise estimate:

$$
\Delta T=18.7 \max \left(\frac{L_{P C}-38.9}{26.8},-1\right)+19.5
$$


These estimates are practical and could be used to predict that a $25 \mathrm{~m}$ long air-cooled chiller plant compound is estimated to result in a $5^{\circ} \mathrm{C}\left(10^{\circ} \mathrm{C}\right)$ average (most affected) temperature rise. However, their application needs to consider that they are derived from analysis using fixed parameters such as plant compound arrangement, clearance space, orientation, lack of adjacent building, and based on a $10 \mathrm{~m} / \mathrm{s}$ wind speed and for air cooled chiller plant compound having a heat rejection density between 15 and $25 \mathrm{~kW} / \mathrm{m}^{2}$. Consequently, caution would be recommended whilst the extent and limitations of such formulation are investigated.

\section{Conclusions}

This study investigates air-cooled chiller plant compound to establish dominant plant compound and environmental parameters affecting air re-entrainment at the air-cooled chillers. A parametric dataset considering, number of chillers, chiller cooling capacity, manufacturer, wind speed, wind direction, is analysed using Computational Fluid Dynamics (CFD) to predict air re-entrainment.

The analysis of the CFD results highlights different mode of air re-entrainment for calm and wind scenarios, and different chiller plume behaviour for increasing number of chillers in plant compound. The dataset shows highest level of air re-entrainment when the wind is aligned with chiller length and highest wind speed analysed.

The statistical analysis of the dataset highlights that there is a difference in air re-entrainment for different chiller manufacturer at low wind speed, but this difference becomes negligible at higher wind speed. The principal component analysis indicates that the plant compound length is the dominant component and air re-entrainment can be estimated from this parameter using a linear relationship.

The study derives a linear approximation of air reentrainment from the plant compound length, which is deemed to be valid for heat rejection density between 15 and $25 \mathrm{~kW} / \mathrm{m}^{2}$, that could be employed at early concept design to estimate air re-entrainment for chiller selection. Caution in the application of this relationship is recommended whilst its limitation is investigated.

\section{Acknowledgements}

The authors would like to thank Airedale and Aermec UK for providing chiller selection datasheets and technical information and Future Facilities, particularly Louise Hewitt and Mark Fenton, for providing 6SigmaDCX software licenses and supporting information.

\section{References}

Alissa, H., Nemati, K., Sammakia, B., Ghose, K., Seymour, M. and Schmidt, R. (2015). Innovative Approaches of Experimentally Guided CFD Modeling for Data Center. SEMITHERM Symposium, (pp. 176-184). San Jose. doi:10.1109/SEMI-THERM.2015.7100157

Borghei, L. and Khoshkhoo, R. H. (2010). Wind effects on air-cooled condenser performance. Taipei.
Brill, K. G. (2006). 2005-2010 Heat Density Trends in Data Processing, Computer Systems, and Telecommunications Equipment: Perspectives, Implications, and the Current Reality in Many Data Centers. The Uptime Institute Inc.

Bruelisauer, M., Meggers, F., Saber, E., Li, C. and Leibundgut, H. (2014). Stuck in a stackTemperature measurements of the microclimate around split type condensing units in a high rise building in Singapore. Energy and Buildings, $71,28-37$.

Buskirk, D. (2018). Excel: Principal Component Analysis (PCA). Retrieved from Learning Tree International: https://blog.learningtree.com/principalcomponent-analysis/

Chow, T., Lin, Z. and Wang, Q. (2000). Effect of building re-entrant shape on performance of aircooled condensing units. Energy and Buildings, 32(2), 143-152.

CIBSE. (2015). AM11 Building Performance Modelling. London: The Chartered Institution of Building Services Engineers.

Ercoftac. (2018). Wind environment around an airport terminal building. Retrieved from The ERCOFTAC Knowledge Base Wiki: http://qnetercoftac.cfms.org.uk/w/index.php/Abstr:Wind_ environment_around_an_airport_terminal_build ing

Future Facilities. (2018). 6SigmaDCX \& 6SigmaET Computational Fluid Dynamics Validation.

Ge, G., Xiao, F., Wang, S. and Pu, L. (2012). Effects of discharge recirculation in cooling towers on energy efficiency and visible plume potential of chilling plants. Applied Thermal Engineering, 39, 37-44.

Khankari, K. (2018). Optimum Placement for AirCooled Chillers. ASHRAE Jounal 58, 18.

Maulbetsch, J. S., DiFilippo, M. N. and O'Hagan, J. (2011). Effect of Wind on Air-Cooled Condenser Performance. Proceeding of the ASME 2011 International Mechanical Engineering Congress \& Exposition IMECE2011-63157, (pp. 391-396).

World's Top Data Centres (2018). Most Power Consumed $(M W)$. Retrieved from World's Top Data Centres: http://worldstopdatacenters.com/power/ 
Ruiz, J. (2015, June). The Calibrated Data Center: Using Predictive Modeling . Retrieved from Uptime Institute:

https://journal.uptimeinstitute.com/thecalibrated-data-center-using-predictivemodeling/
Uptime Institute Professional Services, LLC. (2012).

Data Centre Site Infrastructure Tier Standard:

Topology. New York: Uptime Institute, LLC.

Table 3: Chiller selections' key parameters

\begin{tabular}{|c|c|c|c|c|c|c|c|}
\hline & Manufacturer & $\begin{array}{c}\text { Cooling } \\
\text { Capacity }\end{array}$ & Width, W & Length, L & Height, H & $\begin{array}{c}\text { Input } \\
\text { power }\end{array}$ & $\begin{array}{c}\text { Maximum } \\
\text { Airflow }\end{array}$ \\
\hline A $/ 250 \mathrm{~kW}$ & A & $250 \mathrm{~kW}$ & $2200 \mathrm{~mm}$ & $2626 \mathrm{~mm}$ & $2800 \mathrm{~mm}$ & $54 \mathrm{~kW}$ & $25 \mathrm{~m}^{3} / \mathrm{s}$ \\
\hline A $/ 750 \mathrm{~kW}$ & A & $750 \mathrm{~kW}$ & $2200 \mathrm{~mm}$ & $7154 \mathrm{~mm}$ & $2800 \mathrm{~mm}$ & $171 \mathrm{~kW}$ & $76 \mathrm{~m}^{3} / \mathrm{s}$ \\
\hline A $1500 \mathrm{~kW}$ & A & $1500 \mathrm{~kW}$ & $2200 \mathrm{~mm}$ & $12814 \mathrm{~mm}$ & $2800 \mathrm{~mm}$ & $445 \mathrm{~kW}$ & $139 \mathrm{~m}^{3} / \mathrm{s}$ \\
\hline B/250kW & B & $264 \mathrm{~kW}$ & $2200 \mathrm{~mm}$ & $5160 \mathrm{~mm}$ & $2450 \mathrm{~mm}$ & $85 \mathrm{~kW}$ & $36 \mathrm{~m}^{3} / \mathrm{s}$ \\
\hline B/750kW & B & $762 \mathrm{~kW}$ & $2200 \mathrm{~mm}$ & $9520 \mathrm{~mm}$ & $2450 \mathrm{~mm}$ & $236 \mathrm{~kW}$ & $72 \mathrm{~m}^{3} / \mathrm{s}$ \\
\hline B/1500kW & B & $1519 \mathrm{~kW}$ & $2200 \mathrm{~mm}$ & $17850 \mathrm{~mm}$ & $2450 \mathrm{~mm}$ & $459 \mathrm{~kW}$ & $134 \mathrm{~m}^{3} / \mathrm{s}$ \\
\hline
\end{tabular}
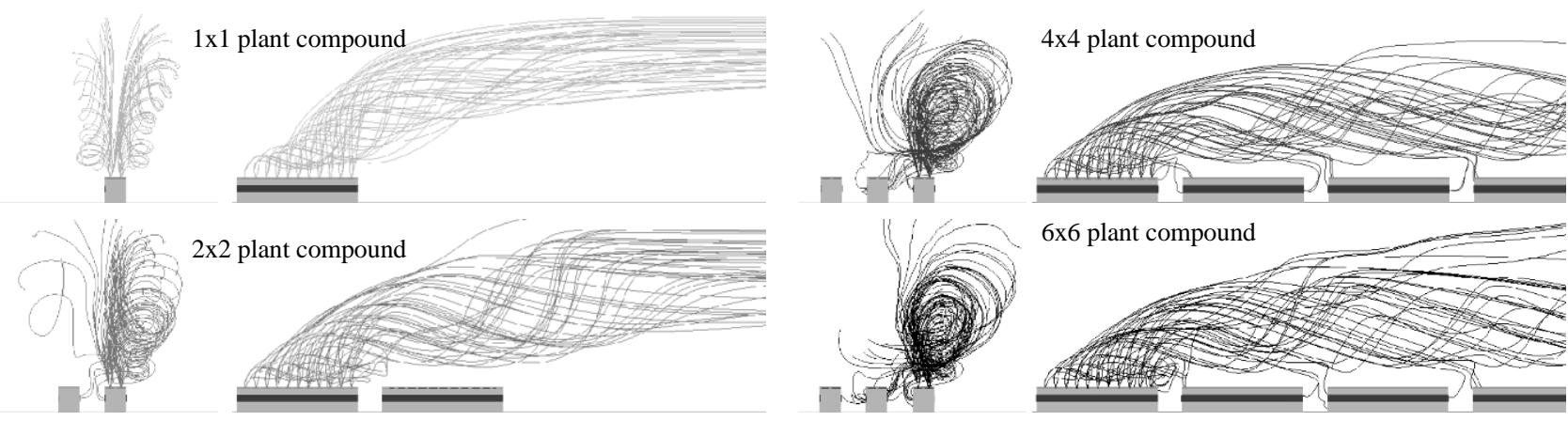

6x6 plant compound

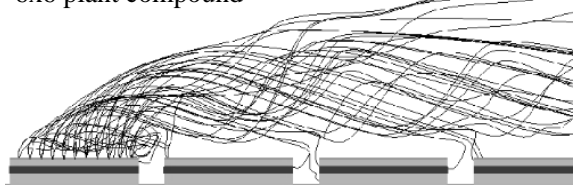

Figure 9: Chiller discharge airflow pattern for chiller A/1500kW in 1x1, 2x2, 4x4 and $6 \times 6$ plant compound under wind direction $1,5 \mathrm{~m} / \mathrm{s}$ wind speed. 